The next step will be to find out whether Tlc has functional homologues in other vertebrate species. Although it is similar in sequence to members of the mammalian sFRP family of Wnt antagonists, none of these has yet been shown to be expressed in the mammalian equivalent of the ANB. However, mutations in other Wnt antagonists, such as the mouse dickkopf gene, can cause loss of telencephalic structures. Taken together with the latest discovery, this indicates that studying the role of Wnt antagonism puts us on track for elucidating the molecular basis of telencephalic induction.

Heather Wood

(2) References and links ORIGINAL RESEARCH PAPER Houart, C. et al Establishment of the telencephalon during. gastrulation by local antagonism of Wnt signalling. Neuron 35, 255-265 (2002)

FURTHER READING Stern, C. Initial patterning of the central nervous system: how many organizers? Nature Rev. Neurosci. 2, 92-98 (2001)

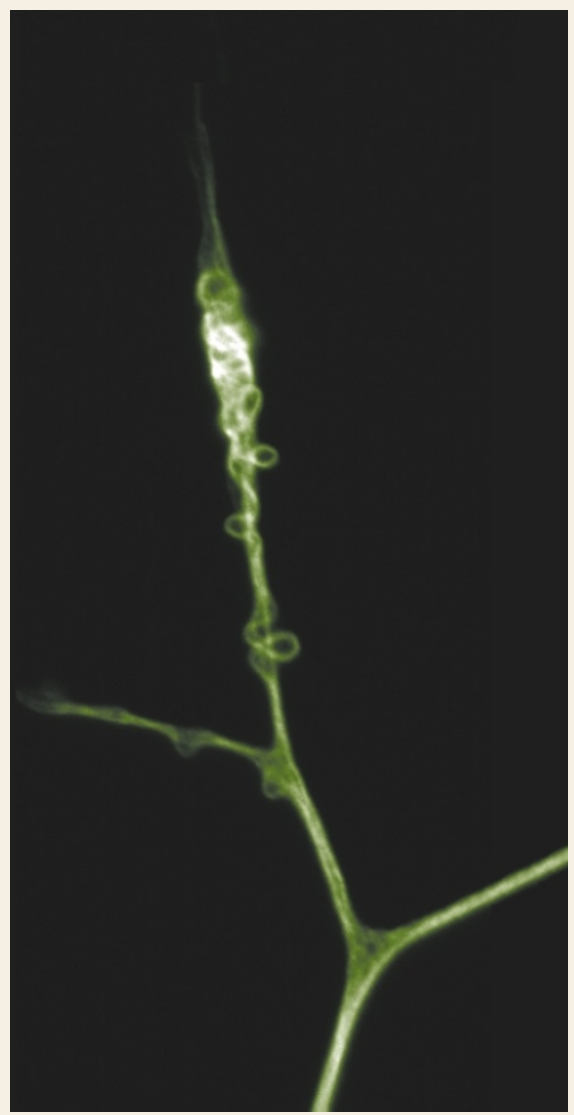

Coiling of microtubule bundles in a retracted axon. Courtesy of Peter Bars, Drexel University College of Medicine, Philadelphia, Pennsylvania, USA.

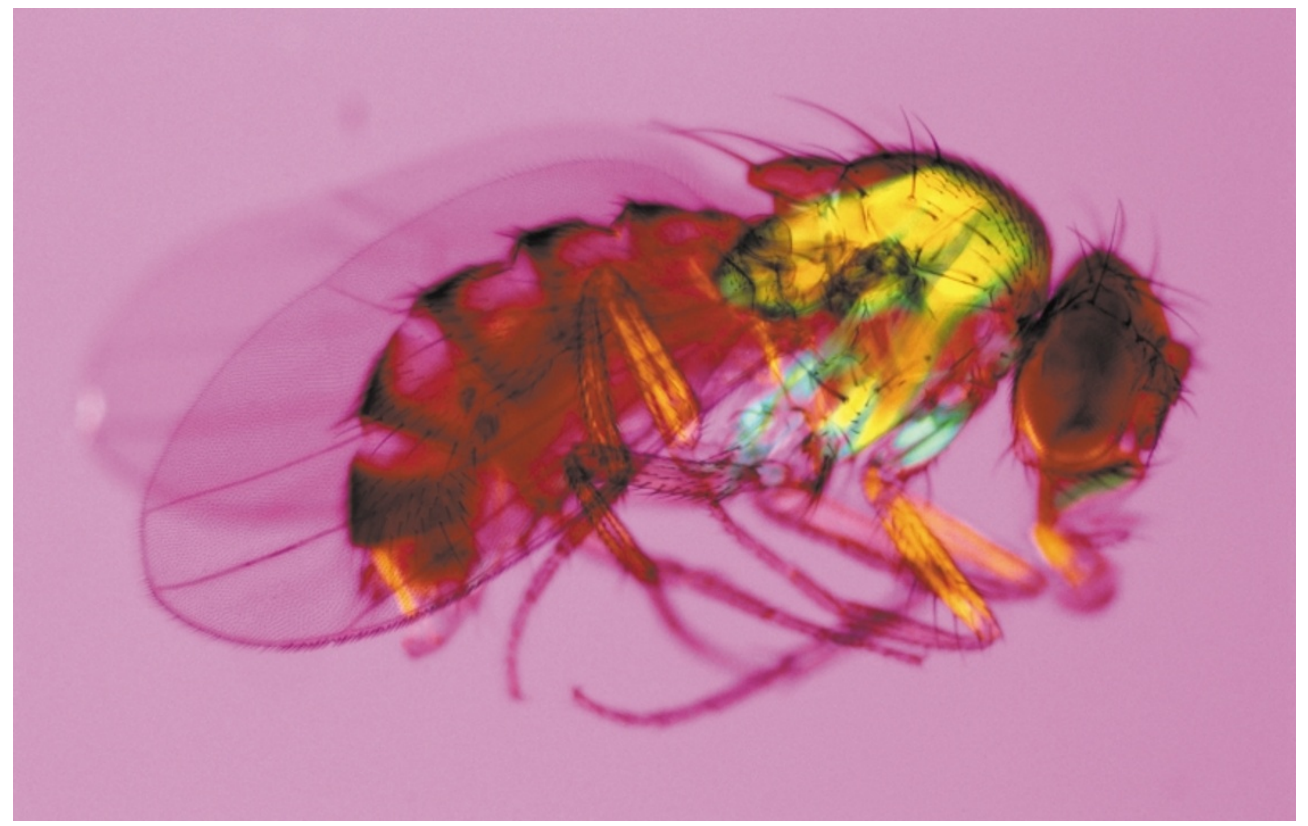

DEVELOPMENT

\title{
Sox, flies and neurogenesis
}

The Sox genes encode a family of DNA-binding proteins, and they are divided into several subgroups on the basis of sequence homology. The vertebrate group B genes, Sox 1 , Sox 2 and Sox3, are all expressed in the neural tube from the early stages of its development, but their roles in early neural development remain unclear. Now, Buescher et al. and Overton et al. report in Development that the fruitfly Drosophila melanogaster could provide some clues.

In the Drosophila neuroectoderm, the neuronal precursor cells, or neuroblasts, arise from proneural clusters, which are arrayed along three longitudinal stripes on each side of the embryo. From each cluster, one cell is singled out to become a neuroblast, and lateral inhibition prevents the rest from adopting a neuronal fate. The clusters are specified by proneural genes, including achaete (ac). The dorsoventral patterning genes ventral nerve cord defective ( $v$ nd), intermediate neuroblasts defective (ind) and muscle segment homeobox $(m s h)$ confer spatial identity on the ventral, intermediate and lateral clusters, respectively.

In Drosophila, there are two group B Sox genes - SoxNeuro (SoxN) and Dichaete. Dichaete is expressed only in the ventral and intermediate neuroectodermal stripes, and SoxN is expressed throughout the neuroectoderm. Mutations in Dichaete cause a reduction in the numbers of ventral and intermediate neuroblasts, and in these new studies, both groups showed that mutations in SoxN cause specific neuroblasts to be lost from the lateral and intermediate regions. Mutating the SoxNgene in a Dichaete-negative background generated a more severe phenotype in the intermediate and ventral clusters, indicating that Dichaete and SoxN act in a partially redundant manner to control the development of neuroblasts in these domains.
To investigate the precise function of SoxN during neurogenesis, Buescher $\boldsymbol{e t}$ al. asked whether it is required for the formation of proneural clusters. They found that although $a c$ expression was reduced in SoxN mutant embryos, the initial establishment of proneural clusters was largely normal. However, in many clusters, no cells were singled out to become neuroblasts, so SoxN seems to be crucial for this step.

Buescher et al. also examined the interactions of SoxN with the patterning genes $v$ nd or ind. In a mutant strain in which Sox $N$ function was only partially lost, the additional loss of one copy of $v n d$ or ind increased the severity of the SoxN mutant phenotype in the ventral and intermediate clusters, respectively. However, mutations in SoxN did not affect the expression of either gene. The authors concluded that SoxN might interact with Vnd and Ind to modulate their activities, but not by controlling their expression.

Much of the molecular machinery that regulates the processes of neural induction and neuronal specification has been conserved throughout evolution, so it is likely that at least some aspects of Sox gene function have been conserved too. Homologues of the proneural genes and $v n d$ and ind have been found in vertebrates, and these genes should provide a good starting point for further investigations into the roles of the group B Sox genes in neural development.

Heather Wood

69) References and links

ORIGINAL RESEARCH PAPER Buescher, M. et al. Formation of neuroblasts in the embryonic central nervous system of Drosophila melanogaster is controlled by SoxNeuro. Development 129 , 4193-4203 (2002) | Overton, P. M. et al. Evidence for differential and redundant function of the Sox genes Dichaete and SoxN during CNS development in Drosophila. Development 129, 4219-4228 (2002) FURTHER READING Bertrand, N. et al. Proneural genes and the specification of neural cell types. Nature Rev. Neurosci. 3 , 517-530 (2002) 